\title{
KOSTEN-EFFEKTIVITÄTS-ANALYSEN \\ ALS GRUNDLAGE PRAKTISCHER UMWELTPOLITIK
}

\author{
Alfred VOB, Albrecht Reuter \\ Universität Stuttgart \\ Institut für Kernenergetik \\ und Energiesysteme (IKE)
}

1 Einleitung

Negative Effekte ökonomischer Aktivitäten auf die Umwelt und Natur, oder anders ausgedrückt, Probleme im Zusammenhang mit der Nutzung der offentlichen Güter Natur und Umwelt, sind ja keineswegs neu, sondern sie haben die Entwicklung der Menschheit seit jeher begleitet. So $z$. B. finden sich bereits in der römischen Gesetzgebung Vorschriften zur Reinhaltung der Gewässer. Die Nutzung von Energie in ihren verschiedenen formen war dabei immer eine der Hauptquelien der Natur- und Umweltbelastung.

Das gegenwätig verstärte öfentliche und politische Interesse an Uwweltfragen steht zweifelsohne in einem engen Zusammenhang mit dem sogenannten Waldsterben oder besser gesagt mit den 'neuartigen" Waldschäden, die seit Ende des letzten Jahrhunderts mit grober geographischer Verbreitung und rasch fortschreitend aufgetreten sind.

Trotz einer erheblichen Verstärung der Anstrengungen zur Erforschung der Ursachen der Waldschäden ist bis heute letztlich nicht geklart, welche Faktoren oder Faktorkomplexe und Mechanismen die neuartigen Waldschäden verursachen. Eine Vielzahl von Schadenshypothesen wurden und werden diskutiert und wissenschaftlich untersucht. Sie reichen von den verschiedenen Luft- 
schadstoffen uber die Beteiligung von Viren, Bakterien, Mycoplasmen bis hin zu waldbaulichen Fehlern und Klimaeinflüssen.

Was die Luftschadstoffe angeht, so konzentrierte sich die Ursachendiskussion zunächst darauf, dab die Schadwirkungen vor allew uber den Weg einer Versauerung des Bodens durch Belastung mit Schwefeldioxid über die direkten Wirkungen des Schwefeldioxids auf die pflanzen erfolgt. In jüngster zeit wird nun der Hyphothese, daB Stickoxide und Kohlenwasserstoffe, als Ausgangssubstanzen für sekundäre Schadstoffe wie $0 z$ on und andere Photooxidantien, ein wesentlicher verursachender Faktor für die Waldschäden sein können, verstärkt Aufmerksamkeit geschenkt.

Trotz der noch bestehenden Unsicherheiten hinsichtlich der Ursache-Wirkungsbeziehungen stellt der Forschungsbeirat 'Waldschäden/Luftverunreinigungen" fest, daß Erklärungshypothesen, die von Luftschadstoffen völlig absehen, nicht zu belegen waren und daB Luftschadstoffe und ihre Uwwandlungsprodukte eine we sentliche Ursache der neuartigen Waldschäden sind.

Bei nüchterner Betrachtung des gegenwärtigen Standes des Wis sens uber die Ursachen der neuartigen Waldschäden kommt man nicht uwhin festzustellen, dab die Kenntnisse, die die Wissenschaft der Politik und der Wirtschaft fü die Unsetzung in konkrete rationale Handlungen anbieten kann, wenig brauchbar sind. Insbesondere gibt es keine gesicherten Kenntnisse uber mögliche Toleranzschwellen der Schadstoffe, die als Verursacher der neuartigen Waldschaden angesehen werden.

Auf der anderen Seite weisen die Waldschadenserhebungen auf einen dringenden Handlungsbedarf hin und die politisch Verantwortlichen sahen und sehen sich einew Handiungszwang ausgesetzt, der durch eine bffentliche Meinung verstärkt wird, die davon überzeugt ist, das der deutsche Wald in Gefahr ist.

Deutliche Reduzierungen der Emissionen luftgetragener Schadstoffe erfordern Invesitionen in Milliardenhohe und werden uber 
ihre Kostenwirkungen die Wettbewerbssituation der Energieträger verändern.

In Anbetracht der bestehenden Wissenslücken, der hohen Kosten von UnweltschutzmaBnahmen und des bestehenden politischen Handlungsbedarfs sind rationale Entscheidungshilfen für die Fundierung einer effizienten Umweltschutzpolitik von besonderer Bedeutung. Im folgenden soll am Beispiel von Baden-Württemberg und der Schadstoffe $\mathrm{SO}_{2}$ und $\mathrm{NO}_{x}$ aufgezeigt werden, welchen Beitrag Kosten-Wirksamkeits-Analysen mittels der heute verfügbaren Energiemodelle zur Identifizierung effizienter umweltpolitischer Maßnahmen leisten können. Auf die praktischen Erfahrungen der Einbindung derartiger systemanalytischer Instrumente in den umweltpolitischen Diskussions- und Entscheidungsproze $\beta$ wird am Ende kurz eingegangen.

2 Einige Methodische Anmerkungen

Ziel einer effizienten Umweltpolitik muß es sein, die gewünschten Umweltziele mit einem möglichst geringen volkswirtschaftlichen Aufwand $z u$ erreichen. Voraussetzung hierfür wäre zum einen eine quantitative Vorgabe der Umweltziele, z.B. der gewilnschten oder tolerierbaren Schäden durch oder der Immissionskonzentrationen von luftgetragenen Schadstoffen, sowie zum anderen eine genaue Kenntnis der technischen und anderen Möglichkeiten, ihre Wirkungen und Kosten, zur Erreichung der Umweltziele.

Normalerweise würde man die umweltpolitischen Entscheidungen und Maßnahmen einer effizienten Umweltschutzpolitik mittels einer umfassenden Kosten-Nutzen-Analyse ermitteln, welche den Nutzen, d. h. die Schadensreduktion den Kosten der jeweiligen Maßnahmen gegenüberstelit. In Prinzip ließe sich dabei über eine Grenzkosten- und Grenznutzenbetrachtung auch das wirt- 
schaftlich optimale AusmaB der Reduzierung der Umweltbelastung ermitteln.

Derartig umassende Kosten-Nutzen-Analysen sind aber derzeitig nicht durchfuhrbar, da zum einen die Dosis-Wirkungsbeziehungen der verschiedenen Schadstoffe nicht bekannt sind, und zum anderen sich die Schäden oft gar nicht oder nur unzureichend quantifizieren lassen, wie z. B. ein durch die Waldschäden zurückgehender Erholungswert des Waldes.

Anders als eine Kosten-Nutzen-Analyse erwittelt eine KostenWirksankits-Analyse die Kosteneffektivität alternativer MaBnahmen oder Technologien zur Reduzierung der Umweltbelastung, worunter $z$. B. der Kostenaufwand je nicht emittierter Schadstoffeinheit $z u$ verstehen ist. Für die später betrachteten Maßnahmen zur Minderung der $\mathrm{SO}_{2}-$ und $\mathrm{NO}_{x}^{-E m i s s i o n e n ~ a u s ~ e n e r-~}$ gietechnischen Anlagen läBt sich die Kosten-Wirksamkeit mittels der spezifischen Minderungskosten, definiert als Kosten zur Vermeidung des AusstoBes eines $\mathrm{kg} \mathrm{SO_{2 }}$ oder NO ${ }_{x}$ beschreiben. Ziel einer Kosten-Wirksamkeits-Analyse ist es damit, aus den vielfältigen technischen und organisatorischen Möglichkeiten zur Reduzierung von Schadstoffemissionen diejenigen auszuwählen, die eine angestrebte Minderung der Emissionen mit dem geringsten Kostenaufwand erreichen. Eine Reihung alternativer Emissionsmiderungsmaßnahmen nach steigenden spezifischen Minderungskosten ergibt dann eine Gesamtkostenfunktion dieses Schadstoffes. Diese beschreibt den kostenoptimalen, d. h. kosteneffizienten Weg zur Minderung des Ausstoßes dieses Schadstoffes. In der praktischen Anwendung dieser wethodisch einfachen Vorgehensweise treten jedoch dann Schwierigkeiten auf, wenn die spezifischen Minderungskosten einer Maßnahme zeitvariabel sind, oder wenn eine Maßnahwe simultan die Emissionen verschiedener Schadstoffe reduziert.

Uw die komplexen Zusammenhänge des Energieversorgungssystems und den von ihm ausgehenden Emissionen Rechnung zu tragen, werden Kosten-Wirksameits-Analysen zur Emissionsminderung in der Regel mit der Hilfe von Simultations-oder Optimierungsmo- 
dellen des Energiesystems durchgefüht. Simulationsmodelle erlauben dabei in der Regel eine detailierte Abbildung des Energieversorgungssystems, während Optimierungsmodelle eine direkte Ermittiung der Gesamtkostenfunktion ermöglichen sowie die durch die Kosten der Emissionsminderung induzierten Substitutionseffekte zwischen den Energieträgern und Energietechniken miterfassen. Im folgenden werden nun die Ergebnisse einer Kosten-Wirksameits-Analyse von Maßnahmen zur Reduzierung von $\mathrm{SO}_{2}-$ und $\mathrm{NO}_{x}{ }^{-\mathrm{Em}}$ issionen erläutert.

3 Kosteneffiziente $\mathrm{SO}_{2}$ - und $\mathrm{NO}_{x}$-Emissionsminderung in BadenWüttemberg

Die Landesregierung von Baden-Wuittemberg hat in den Jahren 1983 bis 1986 drei Kommissionen beauftragt, ein gleichermaßen die Uwwelt- und ökonomischen Belange berücksichtigendes Konzept fur die Reduktion der $\mathrm{SO}_{2}-$ und $\mathrm{NO}_{x}{ }^{-E m i s s i o n e n}$ aus energietechnischen Anlagen in Baden-Würtemberg zu erarbeiten [1, 2, 3]. In den Kommissionen haben die betroffenen Ressorts der Landesregierung, die Regierungspäsidien, die Anlagenbaufirmen, die betroffenen Wirtschaftsbereiche sowie Vertreter der Wissenschaft witgearbeitet. Gemäß Aufgabenstellung mußte es das primäre $Z$ iel der Kommission sein, die einzelnen denkbaren Maßnahmen zur Minderung der $\mathrm{SO}_{2}-$ und $\mathrm{NO}_{x}$-Emissionen bei den verschiedenen Emittentengruppen systematisch zu untersuchen und entsprechend ihrer emissionsreduzierenden Wirkung und ihrer Kosten zu klassifizieren. Dabei wurden die Emissionsminderungsmaßnahmen für die groben Feuerungsaniagen (teistung $>50 \mathrm{MW}_{\mathrm{th}}$ ) anlagenindividuell untersucht, während fur die große Zahl kieiner Anlagen typische MinderungsmaBnahmen ermittelt wurden. Neben einer detaillierten Analyse der Emissionsminderungstechniken beruhte die durchgeführte Kosten-Effektivitäts-Analyse auf einer modeligestutzen Abschätzung der Wirkungen und Kosten alternativer Maßnahmen oder Maßnahmenkombinationen für den Zeitraum bis 1995 . 
Um die Wirkungen und Kosten von MaBnahmen zur Minderung der $\mathrm{SO}_{2}$ - und $\mathrm{NO}_{x}-\mathrm{Emissionen}$ uber diesen Zeitraum sinnvoll ermitteln zu können, war eine Referenzentwicklung des Energieverbrauchs und der daraus resultierenden Emissionen als Ausgangsund Vergleichsbasis vorzugeben. Die wesentlichen dafur zugrundegelegten Rahmenannahmen waren, daB

- das Bruttoinlandsprodukt um 2 X/a wächst,

- der Stromverbrauch in Baden-Wurttemberg bis zum Jahr 1995 linear it einer durchschnittichen Wachstumsite von

3 s/a zunimint,

- die Energiepreisrelationen im wesentlichen erhalten bleiben und

- die Kohleabnahmeverpflichtungen entsprechend den Vereinbarungen it dew deutschen steinkohlebergbau erfullt werden.

Die Referenzentwicklung darf dabei nicht als Prognose - also als Anspruch, die tatsachlich eintretende Entwicklung vorherzusagen - miBverstanden werden. Sie ist nur eine der notwendigen quantitativen Annahmen, um uberhaupt quantitative Aussagen hinsichtich der Kosten und Wirksamkeit machen zu können.

In Abbildung 1 ist die Ausgangssituation bezüglich der Emission der hier interessierenden Schadstoffe $\mathrm{SO}_{2}$ und $\mathrm{NO}_{x}$ nach den verschiedenen Emittentengruppen in Baden-Wurttemberg dargestelit.

An den gesamten $\mathrm{SO}_{2}$-Emissionen von $223 \mathrm{kt}$ hat die Industrie und der Kraftwerkssektor einen Anteil von 39 bzw. 35 , während ihre Anteile an Energieverbrauch bei nur 24 bzw. $16 x$ liegen. Grobter NO${ }_{x}^{-E m i t t e n t ~ i s t ~ d e r ~ V e r k e h r s s e k t o r ~ m i t ~ e i n e m ~ A n t e i l ~}$ von $68 \mathrm{x}$ an den Emissionen in Höhe von $350 \mathrm{kt}$. 


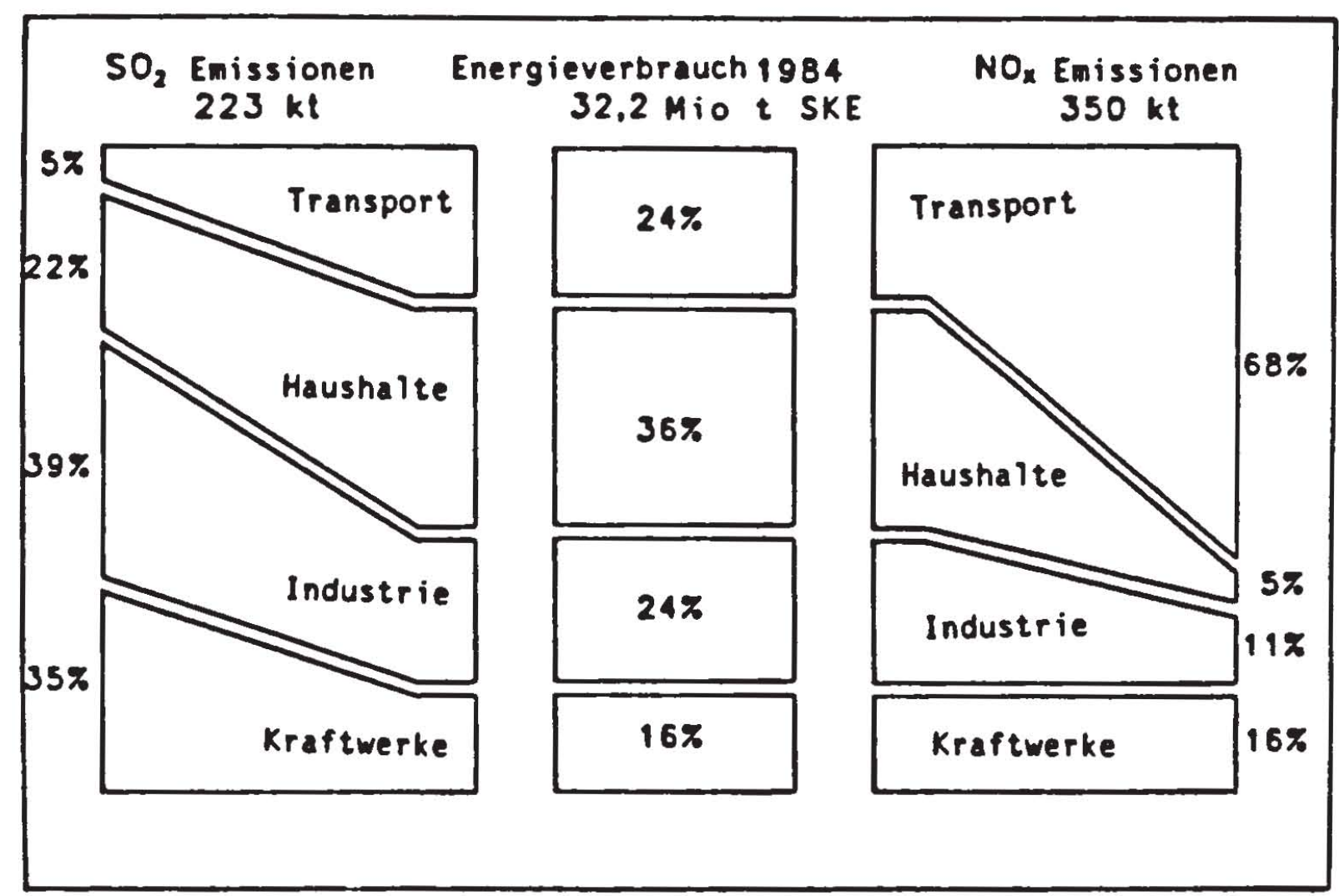

Abbildung 1: Energieverbrauch und Emissionssituation in BadenWürttemberg

Kraftwerkssektor

Ausgehend von einem 3 xigen Stromverbrauchszuwachs steigt die Strombereitstellung von 43000 GWh/a in 1983 auf 63000 GWh im Jahr 1995 an.

Aufgrund auslaufender Stromimportverträge nimt im Jahre 1985 die Stromerzeugung in Baden-Wurttemberg stark zu. Über den gesamten Zeitraum betrachtet wächst der Anteil der Kernenergie an der Stromerzeugung durch die Inbetriebnahme der Kernkraftwerke Phillipsburg II und Neckarwestheim II auf fast $60 \mathrm{X}$ an.

Aber auch die Stromerzeugung aus Kohlekraftwerken nimmt, unter anderew wegen der Kohleabnahmeverpflichtungen, bis 1988 um 50 \% zu. 


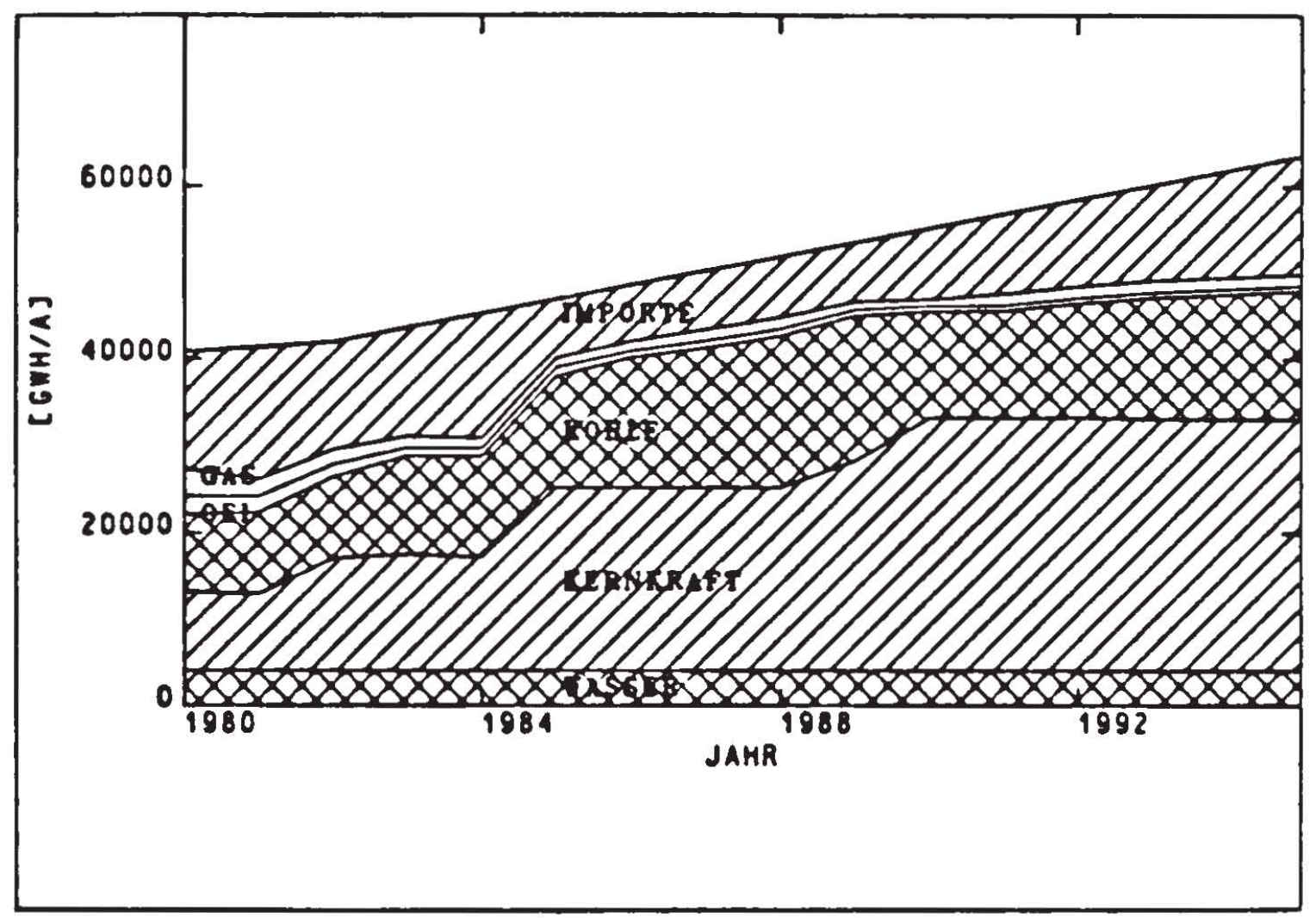

Abbildung 2: Nettostromerzeugung der offentlichen Kraftwerke in Baden-Wlirttemberg

Von den Komissionen wurden eine Vielzahl von MaBnahmen zur Reduktion der $\mathrm{SO}_{2}{ }^{-}$und $\mathrm{NO}_{x}-\mathrm{Emissionen}$ untersucht. Da sind zunachst einal die Brennstoffentschwefelung oder genauer gesagt, der Einsatz schwefelarmer oder schwefelarmerer Kohle und die Steuerung des Verbrennungsprozesses, die sogenannten Primärmaßnahmen zur Minderung der Stickoxidentstehung zu nennen. Dann die Rauchgasreinigung wit den verschiedenen teils erprobten oder noch in Entwicklung befindichen Verfahren zur Minderung von $\mathrm{SO}_{2}$ - und $\mathrm{NO}_{x}-\mathrm{Em}$ issionen. Neben diesen sehr wirksamen Verfahren der Rauchgasreinigung lassen sich emissionsmindernde Effekte aber auch noch durch ein gezieltes Brennstoffmanagement, 2. B. durch einen bevorzugten Einsatz schwefelarmerer Kohle in Kraftwerksblocken, die noch keine Rauchgasentschwefelungsanlagen haben oder durch eine Brennstoffsubstitution (z. b. eine Erhöhung der verfeuerten Erdgasmenge zu Lasten von schwerem Heiz81), erreichen. Aber auch durch ein die Uwweltberange berucksichtigendes Kraftwerkseinsatzmanagement lassen sich begrenzte Enissionswinderungen erreichen. Zu nennen ist 
hier ein bevorzugter Einsatz von Kraftwerken, die bereits mit einer Rauchgasentschwefelungsanlage ausgerustet sind, soweit dies technisch und von der Lastverteilung her möglich ist.

Abbildung 3 zeigt die Entwicklung der $\mathrm{SO}_{2}$-Emissionen aus öffentlichen Kraftwerken in Baden-Wüttemberg für alternative Annahmen über durchgefürte Minderungsmaßnahmen bis zum Jahr 1995. Die obere durchgezogene Linie beschreibt die Emissionsentwicklung, wenn keinerlei Maßnahmen eingeleitet würden. Der Anstieg der $\mathrm{SO}_{2}$-Emissionen von 80 auf $120 \mathrm{Tsd} t / \mathrm{a}$ bis 1988 resultiert dabei aus der bereits angesprochenen Zunahme der Stromerzeugung in Kohlekraftwerken.

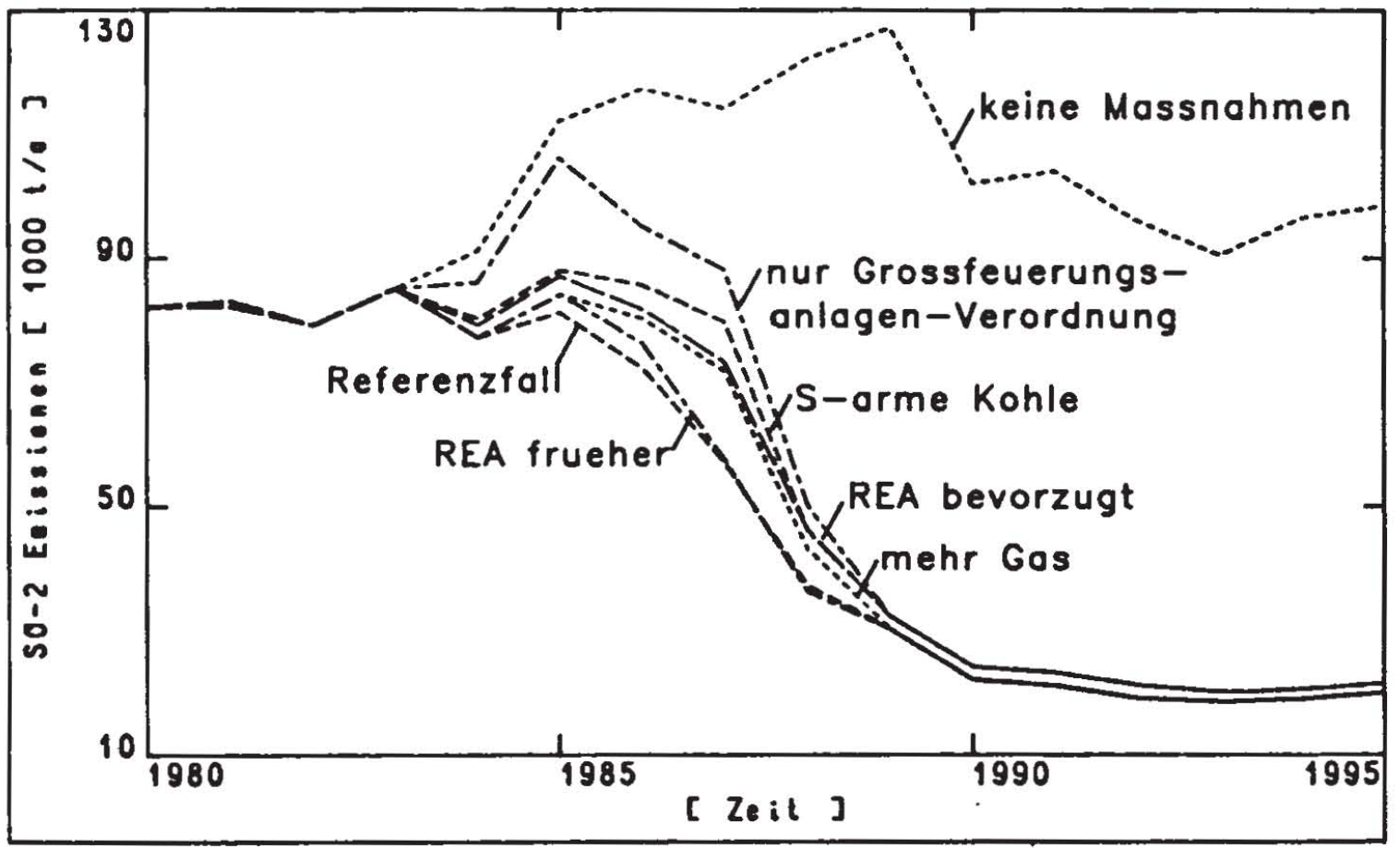

Abbildung 3: Verschiedene Maßnahmen zur Reduktion der $\mathrm{SO}_{2}{ }^{-t i m i s-}$ sionen aus Kraftwerken in Baden-Wurttemberg

Die Realisierung der in der Großfeuerungsanlagenverordnung vorgeschriebenen Grenzwerte fü die $\mathrm{SO}_{2}$-Ewissionen würde nach 1988 die Emissionen drastisch reduzieren und zwar auf ein Niveau von etwa $25 \%$ der derzeitigen Emissionen, trotz eines Anstiegs der Stromerzeugung in fossilen Anlagen. Die in der 
Grobfeuerungsanlagenverordnung verlangten Maßnahmen können aber eine weitere Zunahme der Emissionen im Zeitraum bis 1988 nicht vermeiden.

Die emissionsmindernden Wirkungen einer Reihe von Maßnahmen, die sofort zu einer Emissionsentlastung beitragen können, und die ein vertretbares Kosten-Effektivitäts-Verhältnis aufweisen - hierauf wird später noch zurückzukomen sein - sind in dieser Abbildung auch dargestellt.

Da ist zunächst einmal der optimierte Einsatz schwefelärmerer Kohle zu nennen. Der wittlere Schwefelgehalt heimischer Vollwertkohle liegt bei ca. 0,9 bis 0,95 X Schwefel, bezogen auf SKE. Die Elektrizitätsversorgungsunternehmen in Baden-Wüttemberg beziehen aber teilweise deutsche Steinkohle, deren Schwefelgehalt niedriger, 2 . B. nur $0,8 \%$ ist. Wird nun diese schwefelärmere Kohle, soweit dies technisch möglich ist, in Kraftwerken verfeuert, die noch keine Rauchgasentschwefelung haben, so lassen sich die $\mathrm{SO}_{2}-\mathrm{E}$ issionen deutlich reduzieren, wobei sich für den Zeitraum 1984 bis 1988 Minderemissionen von etwa $35000 \mathrm{t}$ ergeben. Weitere Emissionsminderungen lassen sich durch einen bevorzugten Einsatz der Kraftwerke erreichen, die mit einer Rauchgasentschwefelungsanlage - sei es auch nur einer ersten Stufe - ausgerustet sind. Auch der verstäkte Einsatz von Gas zu Lasten von schwerem Heizbl in Kraftwerken wit Mischfeuerungen trägt in begrenzten Umfang zur Emissionsminderung bei.

Gröbere Effekte in Hinblick auf eine Senkung des $\mathrm{SO}_{2}$-Ausstobes sind von einer fruheren Inbetriebnahme der Rauchgasentschwefelungsanlagen, a 13 von der Grobfeuerungsanlagenverordnung (1.7.1988) und den Genehmigungsauflagen gefordert, zu erwarten. Die angestrebte fruhere Inbetriebnahme der rauchgasent schwefelungsanlagen bewirkt eine Minderung der $\mathrm{SO}_{2}$-Emissionen un 24000 t bis zum Jahr 1988.

Alle diese Maßnahmen bewirken eine kurzfristige wirksam werdende Minderung der $\mathrm{SO}_{2}$-Emissionen um etwa $25 \%$ gegenuber der 
Emissionsentwicklung, die sich nur bei der Durchführung der Grobfeuerungsanlagenverordnung ergeben würden. Darüberhinaus wurden noch weitergehende Maßnahmen untersucht, die aber ein ungünstigeres Kosten-Effektivitäts-Verhältnis aufweisen.

In Tabelle 1 sind die Kosten-Effektivitäts-Relationen für einige der untersuchten Maßnahmen zur Minderung der $\mathrm{SO}_{2}$-Emissionen zusammengestelit. Die Entschwefelung der Rauchgase verursacht je nach GröBe und Auslastung der Anlage Kosten in Höhe von 3-6 $\mathrm{DM}$ je $\mathrm{kg}$ nicht emittiertem $\mathrm{SO}_{2}$. Für die frühere Inbetriebnahme der Rauchgasentschwefelungsanlagen sowie für einen bevorzugten Einsatz von Kraftwerken, die bereits mit einer Rauchgasreinigung ausgestattet sind, fallen Kosten in Höhe von 3 bzw. $0,5 \mathrm{DM} / \mathrm{kg} \mathrm{SO} \mathrm{S}_{2}$ an.

Unterstellt man, das ein verstärker Einsatz von schwefelarmer Importkohle zu Lasten heimischer Steinkohle möglich wäre (unter den gegenwärtigen Bedingungen wäre dies nur möglich, wenn die Kohleabnahmeverpflichtungen nicht erfüllt werden müstenl, dann würde sich beim gegenwätigen Preisvorteil der Importkohle ein Kosten-Effektivitäts-Verhältnis von etwa-10 DM/kg SO 2 ergeben.

Im Vergleich zu den vorgenannten Maßnahmen liegen die spezifischen Kosten eines vorgezogenen Erdgaseinsatzes mit 16 DM/kg $\mathrm{SO}_{2}$ und einer Ausrustung von Altanlagen, die nur noch eine geringe Restnutzungsdauer haben, wit $40 \mathrm{OM} / \mathrm{kg} \mathrm{SO}_{2}$ bedeutend höher; sie sind daher wenig effektiv.

Wie bekannt, lassen sich die Stickoxidemissionen aus Kraftwerken einmal durch feuerungstechnische MaBnahmen, die sog. Primärmabnahmen, und zum anderen durch eine Rauchgasreinigung reduzieren. 
Tabelle 1: Kosten-Effektivitats-Relationen von Maßnahmen zur Minderung von $\mathrm{SO}_{2}-\mathrm{Em}$ issionen aus Kraftwerken

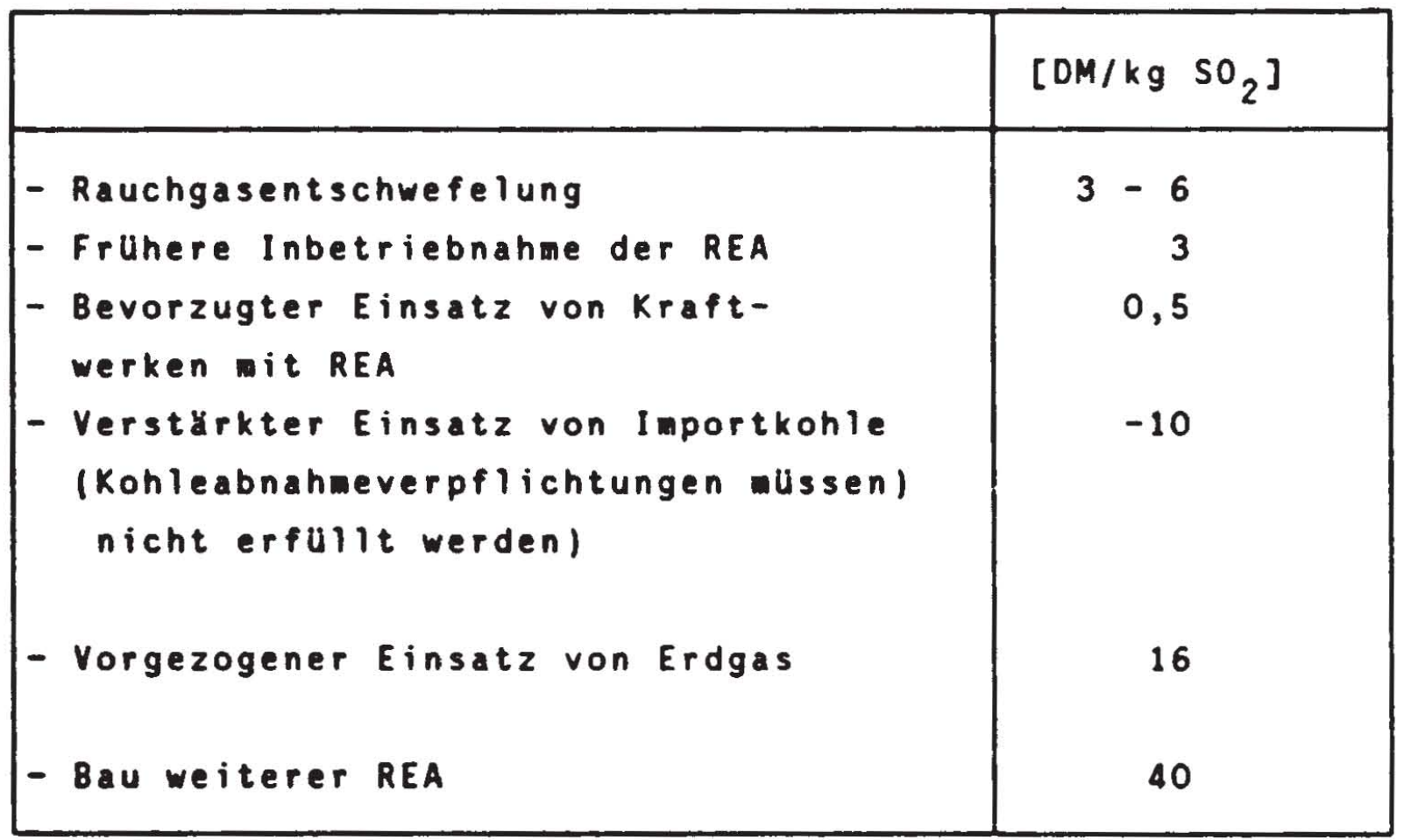

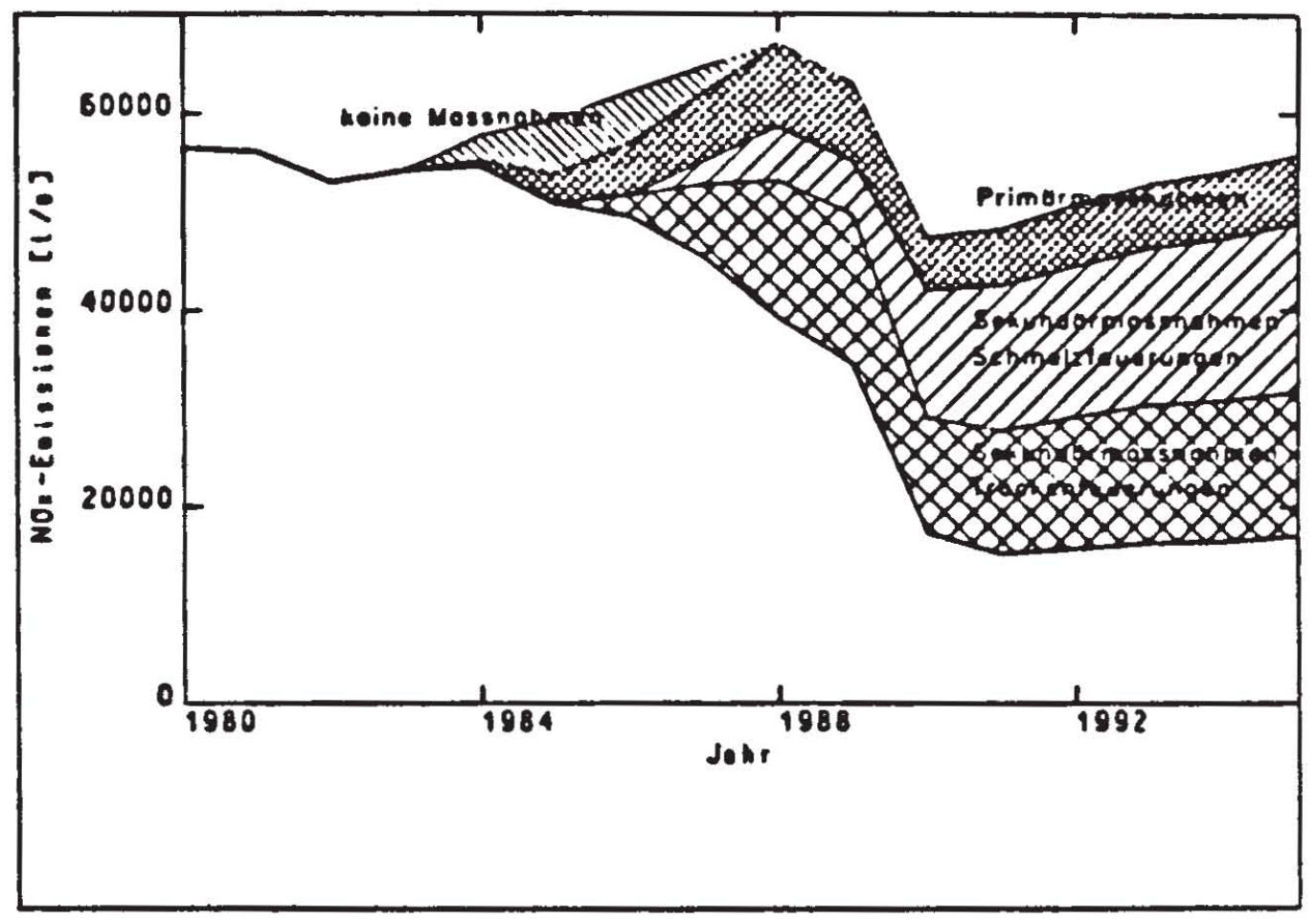

Abbildung 4: NO ${ }^{-E m i s s i o n e n ~ a u s ~ K r a f t w e r k e n ~ i n ~ B a d e n-W u r t t e m-~}$ berg 
Ohne Emissionsminderungsmaßnahmen würde der NO ${ }_{x}^{-A u s s t o B}$ aus öffentlichen Kraftwerken von $55 \mathrm{kt}$ im Basisjahr 1984 auf $76 \mathrm{kt}$ im Jahr 1988 steigen (siehe Abbildung 4). Danach würden die Emissionen aufgrund des verstärkten Kernenergieeinsatzes und eines höheren Anteils moderner Kessel mit Trockenfeuerungen wieder absinken.

Durch Primärmaßnahmen wie der Einbau NO $x$-armer Brenner, Abgasrezirkulation und optimale Brennereinstellung können die $\mathrm{NO}_{x}{ }^{-}$ Emissionen kurzfristig um fast $15 \%$ reduziert werden. Weitere drastische Senkungen der NO ${ }^{-E m i s s i o n e n ~ s i n d ~ d a n n ~ d u r c h ~ E n t-~}$ stickungsanlagen möglich, die die Rohgasbeladungen auf etwa $200 \mathrm{mg} / \mathrm{m}^{3}$ reduzieren. Mit der Installation von Entstickungsanlagen werden die NO ${ }^{-E m i s s i o n e n ~ M i t t e ~ d e r ~ g o e r ~ J a h r e ~ a u f ~ e t w a ~}$ 30 x des Wertes des Jahres 1984 reduziert.

Die Kosteneffektivitäten der verschiedenen No ${ }^{-M i n d e r u n g s m a B-}$ nahmen sind in Tabelle 2 dargestellt.

Tabelle 2: Kosten-Effektivität von $\mathrm{NO}_{x}-$ Emissionsminderungsmaßnahmen in Kraftwerken

\begin{tabular}{|c|c|c|c|}
\hline Maßnahme & $\begin{array}{l}\text { Emissions- } \\
\text { minderung } \\
{\left[10^{3} \mathrm{t}\right]}\end{array}$ & $\begin{array}{l}\text { Jähr } 1 \text { iche } \\
\text { Kosten } \\
{\left[10^{6} \text { DM }\right]}\end{array}$ & $\begin{array}{l}\text { Spezifische } \\
\text { Minderungskosten } \\
\text { (SMK) } \\
{[\mathrm{DM} / \mathrm{kg} \mathrm{NO}]}\end{array}$ \\
\hline keine & - & - & - \\
\hline $\begin{array}{l}\text { Feuerungstechnische } \\
\text { Maßnahmen } \\
\text { Trockenfeuerung }\end{array}$ & 4,93 & 0 & 0 \\
\hline Schmetzfeuerung & 2,03 & 1,95 & 0,96 \\
\hline $\begin{array}{l}\text { DeNO }{ }^{-A n l a g e n} \\
\text { Schmelzfeuerung }\end{array}$ & 17,07 & 79,5 & 4,66 \\
\hline Trockenfeuerung & 14,73 & 101,5 & 6,89 \\
\hline
\end{tabular}


Die spezifischen NO ${ }_{x}^{-M i n d e r u n g s k o s t e n ~ d e r ~ f e u e r u n g s t e c h n i s c h e n ~}$ Maßnahmen liegen unter 1 DM prokg nicht ewittiertem NO ${ }^{\cdot}$ Der Aufwand bei den Sekundärmaßnahmen ist erheblich höher; er liegt zwischen 3 und 8 DM pro kg nicht emittiertem No je nach Kraftwerkstyp und-größe. Im Durchschnitt betragen die NO-Minderungskosten bei den Kraftwerken in Baden-Wurttemberg mit flussigew Ascheabzug 4,6 DM/kg NO und $6,9 \mathrm{DM} / \mathrm{kg}$ NO ${ }_{x}$ bei Anlagen wit trockenew Ascheabzug.

In Abbildung 5 sind die Kostenfunktionen fü die Minderung der $\mathrm{SO}_{2}{ }^{-}$und $\mathrm{NO}_{x}-\mathrm{Em}$ issionen aus Kraftwerken in Baden-wurttemberg dargestellt. Mitte der 90er Jahre läßt sich eine Reduzierung der $\mathrm{SO}_{2}$-Emissionen auf weniger als $20 \mathrm{kt} / \mathrm{a}$ und eine Reduzierung der NO ${ }^{-E m i s s i o n e n ~ a u f ~} 15 \mathrm{kt} / \mathrm{a}$ mit jährlichen Kosten von 330 bzw. 180 Mio DM erreichen. Dies bedeutet eine durchschnittiche Erhöhung der Stromerzeugungskosten in Kohlekraftwerken um ca. 3,7 Dpfg./kWh 1 und bezogen auf den gesamten in Baden-wurttemberg erzeugten Strow entstehen Mehrkosten von 1,1 DPfg./ $W_{\text {el }}$.

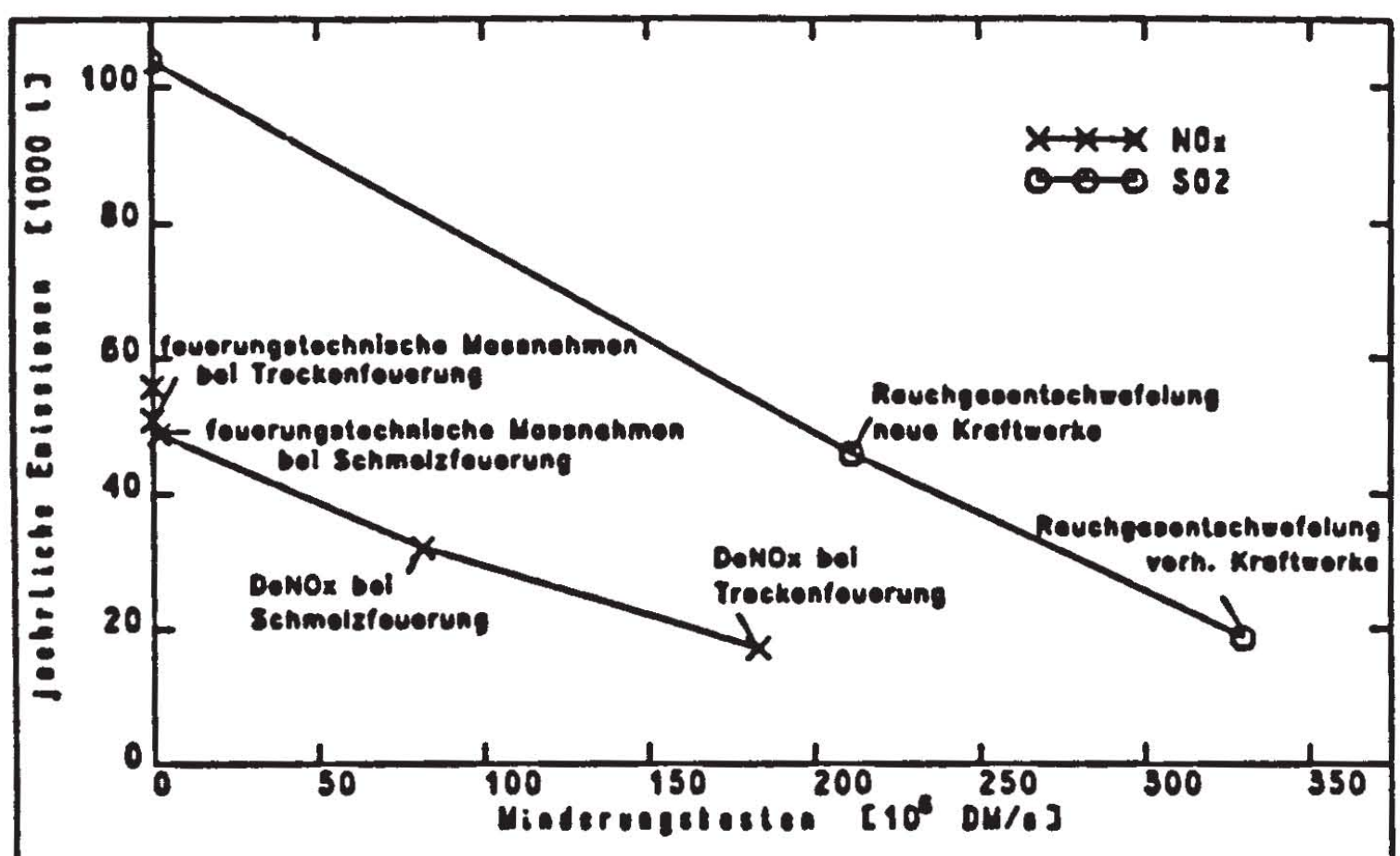

IKE Stuttgort

Abbildung 5: Kostenfunktionen der $\mathrm{NO}_{x}^{-}$und $\mathrm{SO}_{2}{ }^{-E}$ issionsminderung aus Kraftwerken in Baden-Wurttemberg 


\section{Industrie}

Die Industrie ist mit einem Anteil von 39 \% (1984) der größte Emittent von $\mathrm{SO}_{2}$ in Baden-Württemberg, und sie trägt mit etwa 12 X zu den NO ${ }^{-E m i s s i o n e n ~ b e i . ~ D e r ~ u ̈ b e r w i e g e n d e ~ T e i l ~ d e r ~ E m i s-~}$ sionen stammt aus Kessel- und ProzeBfeuerungsanlagen.

In Baden-Wurttemberg gibt es ca. 7800 Betriebe des Bergbaus und des verarbeitenden Gewerbes, die eigene Feuerungsanlagen be treiben. Für die verschiedenen Kategorien von Feuerungsanlagen wurden in Abhängigkeit von der Leistungsgröße die verschiedenen Möglichkeiten der Emissionsminderung systematisch untersucht und analog zum Kraftwerksbereich die erzielbaren $\mathrm{SO}_{2}{ }^{-}$und $\mathrm{NO}_{x}{ }^{-}$ Emissionsminderungen sowie die Minderungskosten ermittelt.

In Abbildung 6 sind die Ergebnisse der Kosten-WirksameitsAnalyse fü den Industriesektor zusammengefaßt dargestellt.

Die Realisierung der in der neuen TA-Luft 86 für Anlagen mit einer Feuerungswärmeleistung bis $2450 \mathrm{MW}_{\text {th }}$ genannten $\mathrm{SO}_{2}-$ und No $x$-Grenzwerte führen in Baden-Württemberg nur zu einer Reduktion dieser Emissionen um 7 bzw. $9 \%$. Die damit verbundenen Gesamtkosten liegen bei $30 \mathrm{Mio} \mathrm{OM} / \mathrm{a}$, da diese Grenzwerte durch Primärmaßnahmen bzw. die Verwendung schwefelärmerer Brennstoffe eingehalten werden können.

Eine weitergehende Minderung der Emissionen ist durch Rauchgasreinigungsmabnahmen, Brennstoffsubstitutionen und die Entschwefelung des leichten Heizöls erreichbar. Die Minderungskosten und Wirkungen dieser Maßnahmen sind in Abbildung 6 für $\mathrm{SO}_{2}$ und $\mathrm{NO}_{x}$ angegeben. Aus der Kostenkurve wird die Verschlechterung der Kosten-Effektivitat bei den verschiedenen Maßnahmen deutlich. 


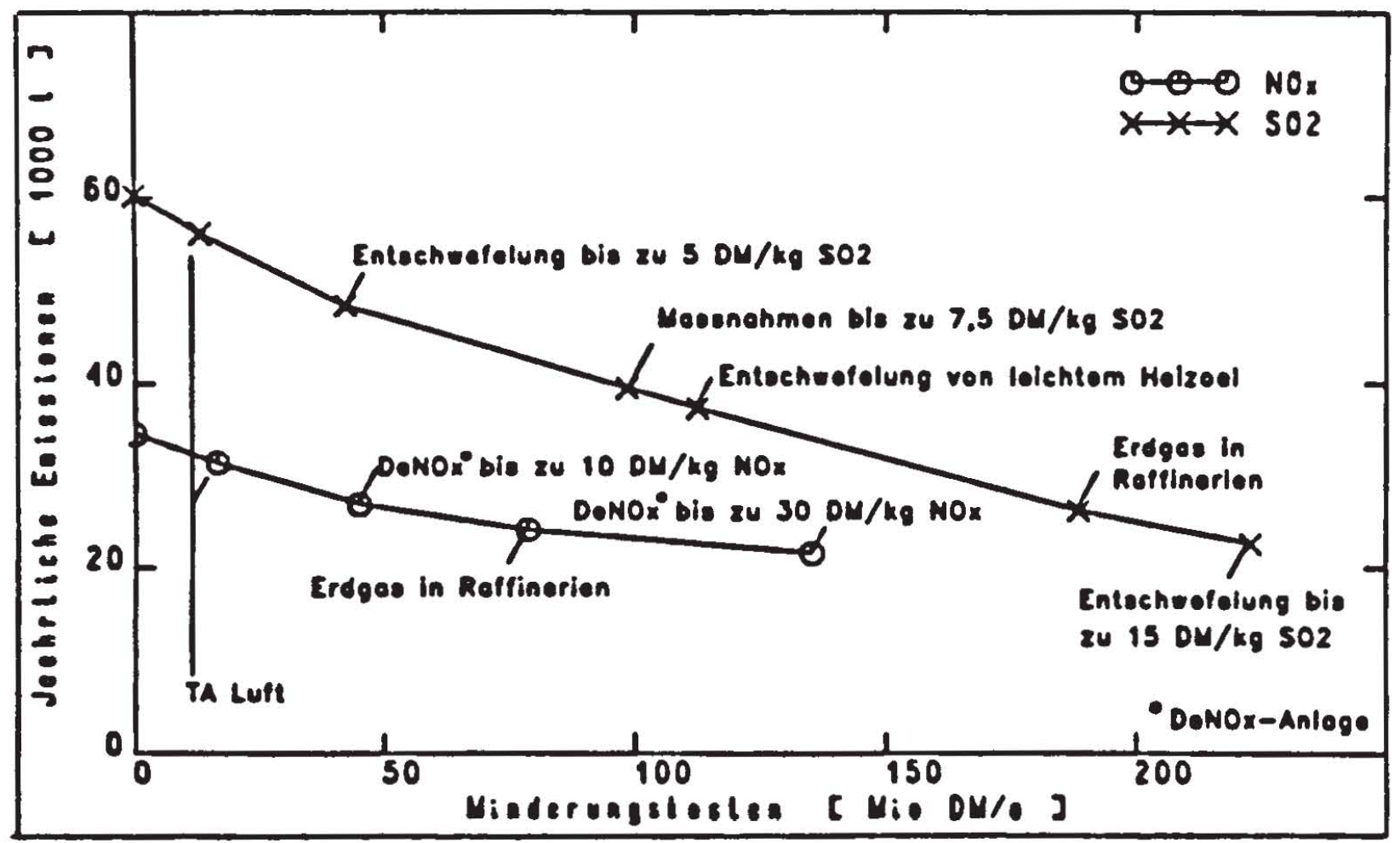

Abbildung 6: Kostenfunktionen der $\mathrm{NO}_{x}^{-}$und $\mathrm{SO}_{2}$-Minderung für den Industriesektor

Alle die hier aufgeführten Maßnahmen können die $\mathrm{SO}_{2}{ }^{-E m i s s i o n e n}$ aus industriellen feuerungsanlagen von 60 auf $22,5 \mathrm{kt} / \mathrm{a}$ bei jährlichen Kosten von 220 Mio OM reduzieren. Eine Reduktion der NO ${ }^{-E m i s s i o n e n}$ von $34 \mathrm{kt} / \mathrm{a}$ a $21 \mathrm{kt} / \mathrm{a}$ liebe sich mit einew Kostenaufwand von $135 \mathrm{Mio} \mathrm{OM} / \mathrm{a}$ erreichen.

Haushalts- und Kleinverbrauchssektor

Der Haushalts- und Kleinverbrauchssektor ist durch eine grobe Zahl kleiner Emissionsquellen gekennzeichnet. Die sinnvollsten Möglichkeiten zur Reduktion der $\mathrm{SO}_{2}-\mathrm{Emissionen}$ bestehen in der 
Entschwefelung des leichten Heizöls, in der Kohlesubstitution und in der Verbesserung der Nutzungsgrade der kleinen Kesselanlagen. NO ${ }_{x}-E m i s s i o n e n ~ l a s s e n ~ s i c h$ durch verschiedene feuerungstechnische Maßnahmen vermindern.

Auch die Emissionsminderungsmöglichkeiten in diesen Sektoren wurden systematisch untersucht, und es wurden, wie bei den zuvor besprochenen Bereichen, Gesamtkostenkurven für eine effiziente Emissionsminderung ermittelt. Hierauf soll aber nicht năher eingegangen werden.

\section{Verkehr}

Der Verkehrssektor ist die Hauptquelle der No ${ }^{-E m i s s i o n e n . ~}$ Trotz einer erwarteten Zunahme der Verkehrsleistung bis 1995 werden die NO ${ }_{x}^{-E m i s s i o n e n ~ a u c h ~ o h n e ~ e m i s s i o n s m i n d e r n d e ~ M a ß n a h-~}$ men nicht weiter ansteigen, da auch weiterhin mit einer Reduktion des spezifischen Kraftstoffverbrauchs und einem weiterhin steigenden Anteil von Diesel-Fahrzeugen, die nur etwa halb soviel ${ }^{N}{ }_{x}$ emittieren wie ungereinigte Otto-Motoren, zu rechnen ist.

Die wesentlichen technischen Möglichkeiten zur Minderung des No ${ }_{x}$-AusstoBes von Otto-Motoren sind der geregelte und ungeregelte Drei-Wege-Katalysator, die Abgasrückührung und der Magermotor. Der geregelte Drei-Wege-Katalysator erreicht dabei Reduktionsgrade von 70 bis $90 \mathrm{x}$, der ungeregelte Katalysator etwa $50 \%$.

Die neuen Enissionsgrenzwerte der EG-Kommission führen bis 1995 nur zu einer Reduktion der NO ${ }_{x}$ Emissionen um $7 \%$. Unterstellt man, daß die von der Bundesregierung gegebenen finanziellen Anreize für abgasgereinigte Kraftfahrzeuge bis zum Jahr 1995 dazu fuhren, daß 50 \% der Altfahrzeuge nachgerustet und der Anteil der Neufahrzeuge mit Katalysator bis 1988 bzw. 1993 auf 
100 \% zunimmt, dann wïrden sich die NO ${ }^{-E m i s s i o n e n ~ d e s ~ V e r-~}$ kehrssektors bis 1995 um $66 \mathrm{kt} / \mathrm{a}, \mathrm{d} . \mathrm{h}$. ca. 30 \% reduzieren. Tabelle 3 listet die erzielbaren Minderungen der einzelnen Maßnahmengruppen, ihre Gesamtkosten sowie die spezifischen Minderungskosten auf.

Tabelle 3: NO -Reduktionen und ihre Kosten im Verkehrssektor $x$

\begin{tabular}{|c|c|c|c|}
\hline MaBnahme & $\begin{array}{l}\text { NO -Emissions- } \\
\times \text { minderung in } \\
1995 \\
{[1000 \mathrm{t} / \mathrm{a}]}\end{array}$ & $\left\{\begin{array}{l}\text { Minderungs- } \\
\text { kosten } \\
{\left[\begin{array}{cc}{ }^{6} & \mathrm{DM} / \mathrm{a}]\end{array}\right.}\end{array}\right.$ & $\begin{array}{l}\text { spez. Minde- } \\
\text { rungskosten } \\
{[D M / k g N O]_{x}}\end{array}$ \\
\hline $\begin{array}{l}\text { Nachrüstung } \\
\text { geregelter und } \\
\text { ungeregelter } \\
3 \text {-Wege-Katalysator } \\
\text { in neuen PKW } \\
1400-2000 \mathrm{ccm} \\
>2000 \mathrm{ccm}\end{array}$ & 7,5 & $\begin{array}{l}215 \\
740\end{array}$ & $\begin{array}{l}15,2 \\
16,6\end{array}$ \\
\hline Gesamt & 66,2 & 1054 & 15,9 \\
\hline
\end{tabular}

Nachdem in den vorangegangenen Ausführungen die Möglichkeiten und Kosten der Reduzierung der $\mathrm{SO}_{2}-$ und $\mathrm{NO}_{x}-\mathrm{Em}$ issionen in den verschiedenen Sektoren diskutiert wurden, ist es nun möglich, eine effiziente Gesamtstrategie der Minderung dieser luftgetragenen Schadstoffe für Baden-Wüttemberg zu entwickeln.

Abbildung 7 zeigt die Kostenfunktion für eine kostenoptimale Reduktion der $\mathrm{SO}_{2}-\mathrm{Em}$ issionen in Baden-Wüttemberg. Die $\mathrm{SO}_{2}{ }^{-}$ Emissionen in Baden-Wurttemberg könnten Mitte der goer Jahre mit jährlichen Kosten von 700 Mio DM um 70 \% gegenüber 1984 reduziert werden. Die effizientesten Maßnahmen sind dabei die 
Installation von Entschwefelungsanlagen in Kraftwerken und großen industriellen feuerungsanlagen.

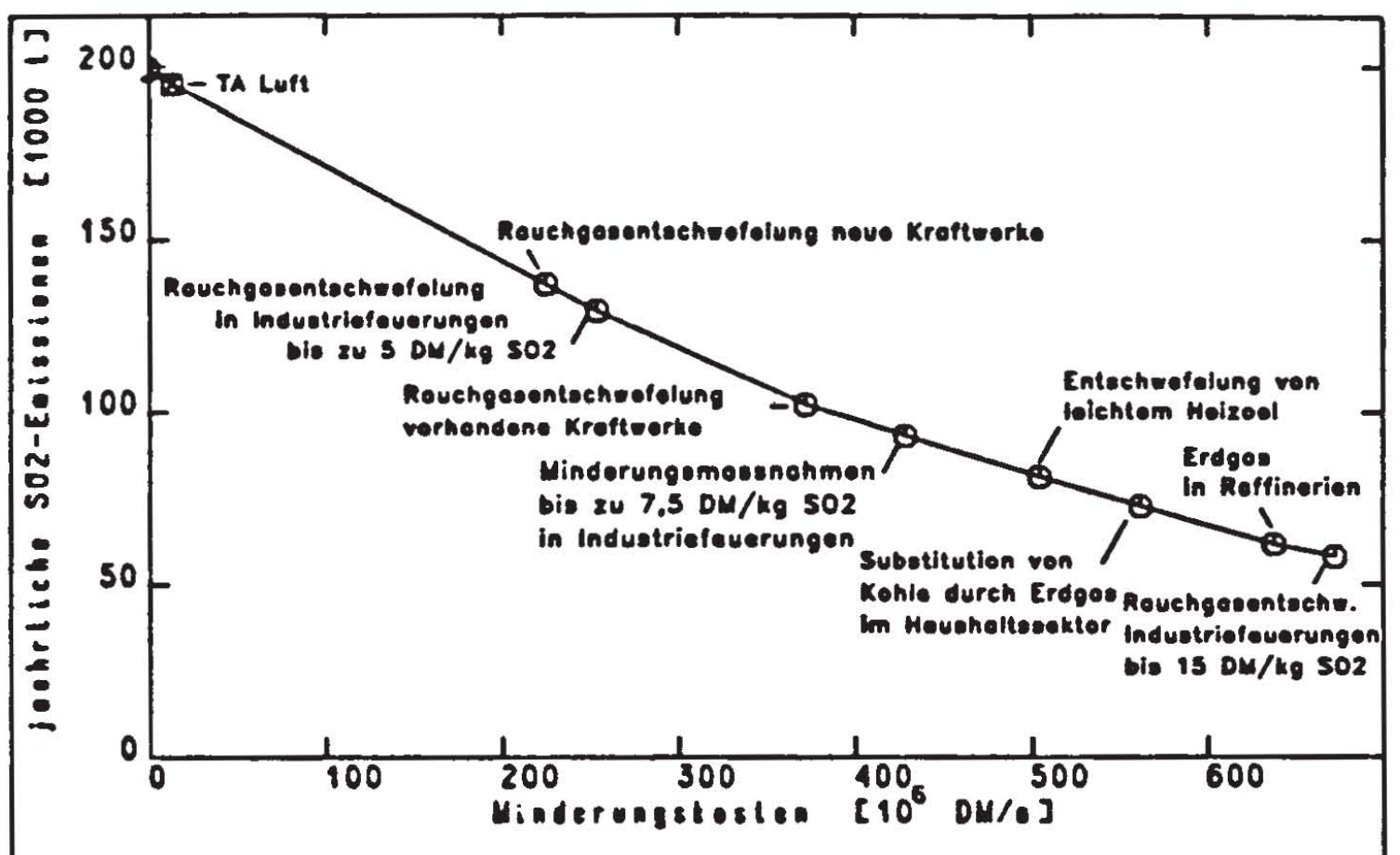

UKE Stuttgart

Abbildung 7: Gesamtkostenkurve der $\mathrm{SO}_{2}$-Minderung in BadenWürt temberg

Ähnlich drastische Reduktionen der NO ${ }^{-E m i s s i o n e n ~ i n ~ B a d e n-~}$ Wurttemberg sind mit den gegenwärtig verfügbaren Techniken nicht erreichbar.

Abbildung 8 zeigt die mit den verschiedenen Maßnahmen erreichbaren $\mathrm{NO}_{x}$-Minderungen und die damit verbundenen Kosten. Mit einem jährlichen Aufwand von 1500 Mio DM wäre lediglich eine Minderung der NO ${ }^{-E m i s s i o n e n ~ u m ~} 37$ x erreichbar. Die Haupt- 
quellen der verbleibenden Emissionen sind die Diesel-Fahrzeuge und die Zementindustrie. Die effizientesten NO ${ }^{-M i n d e r u n g s m a \beta-~}$ nahmen sind die PrimärmaBnahmen bei Feuerungsanlagen sowie die Rauchgasreinigung in Kraftwerken und großen Industriefeuerungs an lagen.

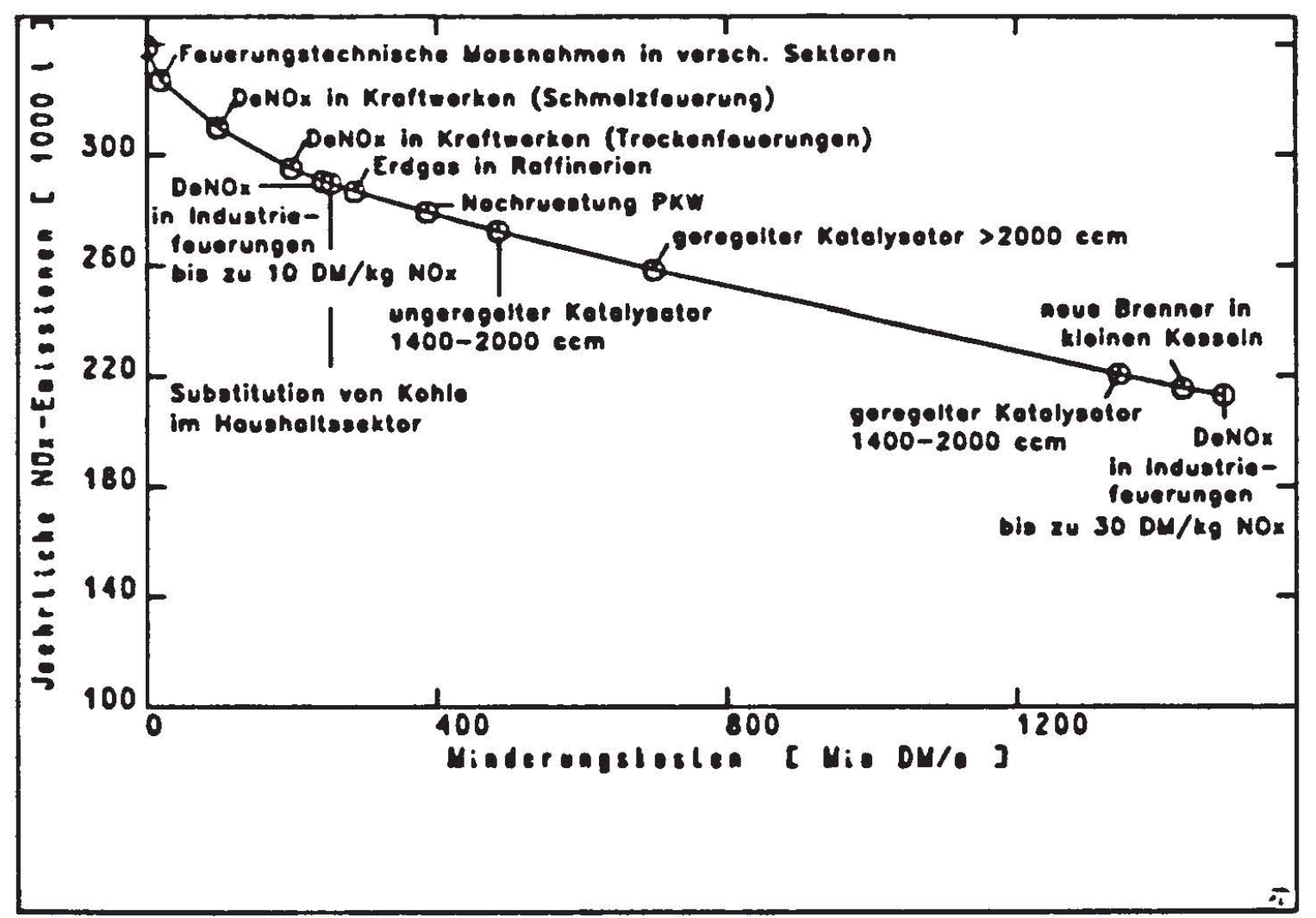

Abbildung 8: Gesamtkostenkurve der NO ${ }^{-M i n d e r u n g}$ in BadenWürt temberg

\section{Zusammenfassende Wertung}

Die vorangegangenen Erläuterungen einer Kosten-WirksamkeitsAnalyse von emissionsmindernden Maßnahmen sollten exemplarisch 
aufzeigen, welche konkreten Informationen und Entscheidungshilfen derartige Analysen für eine effiziente Umweltschutzpolitik liefern können. Es sei noch angemerkt, daß die Ergebnisse der modellgestützten Kosten-Wirksamkeits-Analyse für BadenWüttemberg jeweils direkt in die Diskussionen und Beratungen der verschiedenen, von der Landesregierung eingesetzten Kommis sionen eingebracht wurden. Sie haben zu einer Versachlichung der Diskussison zwischen den unterschiedlichen Interessengruppen beigetragen und waren die wesentliche Grundlage für die Verabschiedung einer Emissionsminderungsstrategie für das Land Baden-Wurttemberg, das über freiwillige Verpflichtungen der jeweiligen Emittentengruppen bereits realisiert worden ist bzw. werden wird.

Angesichts der bestehenden Unsicherheiten über die Ursachen der neuartigen Waldschäden und die Dosis-Wirkungsbeziehungen einzelner Schadstoffe, können umfassende Kosten-WirksameitsAnalysen wertvolle Entscheidungshilfen für eine rationale Umweltpolitik liefern, indem sie aufzeigen, wie bei gegebenem Aufwand der größte emissionsmindernde Effekt erzielt werden $k a n n$.

Ihre Aussagemöglichkeiten sind aber begrenzt, insbesondere wenn es um die Frage der energiewirtschaftlichen und gesamirtschaft lichen Auswirkungen der Kosteneffekte der Minderungspolitiken und die Frage der Vertretbarkeit der Umweltschutzmaßnahmen geht.

In diesem Zusammenhang erschient die Feststeliung wichtig, daß es bis heute an fundierten Analysen der ökonomischen und strukturellen Konsequenzen der bereits verabschiedeten Luftreinhaltemaßnahen fur die Energieversorung und Energiewirtschaft noch fehit. Da es um jährliche Kosten in Milliardenhöhe geht, können sorgfälige Umweltmaßnahmenfolgeabschätzungen auch heute noch von grobem Nutzen sein und zu einer effektiven, sowohl die okonomischen wie ökologischen Belange berücksichtigenden Umwelt-und Energiepolitik beitragen. 


\section{Literaturverzeichnis}

[1] Staatsministerium Baden-Wurttemberg (Hrsg.):

Bericht der Arbeitsgruppe Energiebedarf-Umwelt-Kraftwerksbetrieb',

Stuttgart 1983

[2] Staatsministerium Baden-Wüttemberg (Hrsg.):

Minderung von Stickoxidemissionen aus Kohlekraftwerken in Baden-wlirt temberg,

Stuttgart 1984

[3] Staatsministeirum Baden-Wurttemberg (Hrsg.):

Wirtschaftliche Entwicklung - Unwelt - Industrielle Produktion,

Stuttgart 1986

[4] Voss, A., et a 1.:

Kosten-Effektivitäts-Analyse von Maßnahmen zur Reduzierung von $\mathrm{SO}_{2}$ - und $\mathrm{NO}^{-}$-Emissionen in Ballungsräumen a $\mathrm{m}$ Beispiel der Stadt Stuttgart

Final Report, KFK-PEF

[5] Voss, A.:

Cost and Effectiveness of Reducing Sulphur and Nitrogen Emissions from Power Plants in Baden-Wurttemberg,

ECE-GE.85-43208, Fourth Seminar on the Control of Sulphur and Nitrogen Oxides from Stationary Sources,

Graz, Austria, 12-16 May 1986

[6] Friedrich, R.; Voss, A., Ruff, E.:

Flinf Vorschläge fur reinere Luft,

Energiewirtschaf $1 \mathrm{tiche}$ Tagesfragen, Heft $8,34 . \mathrm{Jg}, 1984$

[7] Friedrich, R.; Mattis, M., Voss, A.:

Entstickung in sechs Schritten,

Energiewirtschaftiche Tagesfragen, Heft $1 / 2,35 . \mathrm{Jg}$, , 1985 